\title{
ON THE DIRICHLET PROBLEMS FOR SYMMETRIC FUNCTION EQUATIONS OF THE EIGENVALUES OF THE COMPLEX HESSIAN *
}

\author{
SONG-YING $\mathrm{LI}^{\dagger}$
}

To Yum-Tong Siu, on his sixtieth birthday

1. Introduction. Let $D$ be a bounded domain in $\mathbb{C}^{n}$, and let $u \in C^{2}(D)$ be a real valued-function. Then the complex Hessian of $u$

$$
H[u](z)=\left[\frac{\partial^{2} u(z)}{\partial z_{i} \partial \bar{z}_{j}}\right]_{n \times n}
$$

is an $n \times n$ hermitian matrix at each point $z \in D$. Let $\lambda(H(u))=\left(\lambda_{1}(z), \cdots, \lambda_{n}(z)\right)$ be all eigenvalues of $H[u](z)$ as a vector in $\mathbb{R}^{n}$. Then the kth elementary symmetric function $\sigma^{(k)}$ is defined as follows:

$$
\sigma^{(k)}(\lambda)=\sum_{i_{1}<\cdots<i_{k}} \lambda_{i_{1}} \cdots \lambda_{i_{k}}
$$

In particular,

$$
\operatorname{det} H(u)=\sigma^{(n)}\left(\lambda(H(u)), \quad \Delta u=\operatorname{tr}(H(u))=\sigma^{(1)}(\lambda(H(u))) .\right.
$$

It was proved in [8] that $\sigma^{(k)}(\lambda)^{1 / k}$ is a concave strictly increasing function on the symmetric convex cone:

$$
\Gamma_{k}=\left\{\lambda \in \mathbb{R}^{n}: \sigma^{(j)}(\lambda)>0,1 \leq j \leq k\right\}
$$

and

$$
\Gamma_{n}=\left\{\lambda \in \mathbb{R}^{n}: \lambda_{j}>0, \quad 1 \leq j \leq n\right\}, \quad \Gamma_{1}=\left\{\lambda: \sum_{j=1}^{n} \lambda_{j}>0\right\}
$$

Also $\Gamma_{k}$ is symmetric in $\lambda=\left(\lambda_{1}, \cdots, \lambda_{n}\right)$, which means that if $\lambda=\left(\lambda_{1}, \cdots, \lambda_{n}\right) \in \Gamma_{k}$, then $\tilde{\lambda}=\left(\lambda_{i_{1}}, \cdots, \lambda_{i_{n}}\right) \in \Gamma_{k}$ where $\left(i_{1}, i_{2}, \cdots, i_{n}\right)$ is any permutation of $1,2, \cdots, n$. We say that $u$ is plurisubharmonic in $D$ if $\lambda(H(u)(z)) \in \overline{\Gamma_{n}}$, for all $z \in D$; we say that $u$ is subharmonic in $D$ if $\lambda(H(u)(z)) \in \overline{\Gamma_{1}}$ for all $z \in D$. We will let $\Gamma$ be a convex cone which is symmetric in $\lambda \in \Gamma$, with vertex 0 so that $\Gamma_{n} \subseteq \Gamma \subseteq \Gamma_{1}$. Let $\mathcal{M}(n, \Gamma)$ be subset of all $n \times n$ hermitian matices $H$ over $\mathbb{C}$ so that $\lambda(H) \in \Gamma$ where $\lambda(H)$ is a vector in $\mathbb{R}^{n}$ being formed by all eigenvalues of $H$. We will consider more general symmetric function than the $k$ th symmetric function $\sigma^{(k)}$ on $\mathcal{M}(n, \Gamma)$. Let $D$ be a bounded domain in $\mathbb{C}^{n}$ with smooth boundary $\partial D$. We say that a real-valued function $u$ is $\Gamma$-subharmonic if $\lambda(H(u)(z)) \in \Gamma$ for all $z \in D$. we will consider the Dirichlet problem for a functional equation:

$$
F(H(u)(z))=\psi(z), \quad z \in D, \quad u=\phi \quad \text { on } \partial D .
$$

\footnotetext{
*Received April 11, 2003; accepted for publication June 12, 2003.

${ }^{\dagger}$ Department of Mathematics, University of California, Irvine, CA 92697-3875, USA; Wuhan University, Wuhan, China (sli@math.uci.edu).
} 
where $F(H)$ can be written as

$$
F(H)=f(\lambda(H)), \quad H \in \mathcal{M}(n, \Gamma),
$$

with $f$ a symmetric function in $\lambda \in \Gamma$. In order that the equation (1.6) is elliptic, we assume that $f(\lambda)$ is a positive, strictly increasing, concave function on $\Gamma$. In other words, we assume that $f$ is positive, concave and satisfies

$$
\frac{\partial f}{\partial \lambda_{j}}>0, \quad 1 \leq j \leq n
$$

In order to prove an existence theorem, we may assume more on function $f$. Let

$$
\psi_{0}=\min \{\psi(z): z \in \bar{D}\} .
$$

Let $\psi^{0}$ be any positive number so that $\psi^{0}<\psi_{0}$. Then we assume that

$$
\lim \sup _{\lambda \rightarrow \lambda_{0}} f(\lambda) \leq \psi^{0}, \quad \lambda_{0} \in \partial \Gamma
$$

and for any compact subset $K$ of $\Gamma$, we assume that

$$
\lim _{R \rightarrow \infty} f\left(\lambda_{1}, \cdots, \lambda_{n-1}, \lambda_{n}+R\right)=\lim _{R \rightarrow \infty} f(R \lambda)=\infty
$$

uniformly for $\lambda \in K$.

It was proved in [8] that

$$
f_{k}(\lambda)=\left(\sigma^{(k)}(\lambda)\right)^{1 / k}
$$

is a symmetric, positive, strictly increasing concave function on $\Gamma_{k}$ satisfying $(1.10)$ and (1.11).

Let $D$ be a smoothly bounded domain in $\mathbb{C}^{n}$, we say that $D$ is $\Gamma$-pseudoconvex if there is $C_{D} \geq 0$ so that $\lambda\left(-H(d)+C H\left(d^{2}\right)\right) \in \Gamma$ on $\partial D$ where $d(z)$ is the distance function from $z$ to $\partial D$.

The existence of a unique classical solution for the Dirichlet problem of symmetric function of the eigenvalues of real hessian matrix of a function $u$ on a domain in $\mathbb{R}^{n}$ was proved by Caffarelli, Nirenberg and Spruck in [8]. Many other problems related to geometric problems were studied by B. Guan and P. Guan [16], P. Guan and X. Ma in [18], P. Guan, C. S. Lin and X. Ma in [19], P. Guan and Y. Li in [17] and J. Urbas in [34] with references therein and many others. The Dirichlet problem for the complex Monge-Ampere equations on strictly pseudoconvex domain in $\mathbb{C}^{n}$ has been studied many authors, we refer to [1], [2], [9], [7], [15], [25],[22], [26] and references therein. The existence of classical plurisubharmonic solution for complex MongeAmpère equations on strictly pseudoconvex domain was proved by Caffarelli, Kohn, Nirenberg and Spruck in [7]. In [15], B. Guan proved the similar results hold on weakly pseudoconvex domain provided the bounday data has plurisubharmonic subsolution. Results on Hörlder continuity were proved by the author in weakly pseudoconvex domain of finity type in [26]. Based on the all above known results, we are proposed to prove the following theorems which are natural generalizations of the known results mentioned above.

THEOREM 1.1. Let $\Gamma$ be a convex symmetric cone in $\mathbb{R}^{n}$ with $\Gamma_{n} \subseteq \Gamma \subseteq \Gamma_{1}$. Let $D$ be a smoothly bounded domain in $\mathbb{C}^{n}$. Let $f$ be a strictly increasing, concave function 
on $\Gamma$ satisfying (1.10) and (1.11). If $\phi \in C^{\infty}(\partial D)$ has an extension $\underline{u} \in C^{\infty}(\bar{D})$ so that

$$
f(\lambda(H(\underline{u}))) \geq \psi(z)+\epsilon, z \in D \quad \text { for some } \epsilon>0
$$

then the Dirichlet problem

$$
f(\lambda(H(u)))=\psi>0 \quad \text { in } \bar{D}, \quad u=\phi \quad \text { on } \partial D
$$

admits a (unique) solution $u \in C^{\infty}(\bar{D})$ with $\lambda(H(u)) \in \Gamma$ on $D$.

THEOREM 1.2. Let $\Gamma$ be a convex symmetric cone in $\mathbb{R}^{n}$ with $\Gamma_{n} \subseteq \Gamma \subseteq \Gamma_{1}$. Let $D$ be a smoothly bounded $\Gamma-$ pseudoconvex domain in $\mathbb{C}^{n}$. Let $f$ be a strictly increasing, concave function on $\Gamma$ satisfying (1.10) and (1.11). Then the Dirichlet problem

$$
f(\lambda(H(u)))=\psi>0 \quad \text { in } \bar{D}, \quad u=\phi \quad \text { on } \partial D
$$

admits a (unique) solution $u \in C^{\infty}(\bar{D})$ with $\lambda(H(u)) \in \Gamma$ on $D$.

In particular, for $f=\sigma^{(k)}$, we have the following theorem.

TheOREM 1.3. For $1<k \leq n$, the Dirichlet problem

$$
\sigma^{(k)}(\lambda(H(u)))=\psi>0 \quad \text { in } \bar{D}, \quad u=\phi \quad \text { on } \partial D
$$

admits a (unique) admissible solution $u \in C^{\infty}(\bar{D})$ provided that

$$
\partial D \text { is connected, and } \sigma^{(k-1)}(\lambda(\mathcal{L}))>0 \text { on } \partial D
$$

where $\mathcal{L}$ is a Levi-form of $\partial D$.

Moreover, when $\phi=$ constant, condition (1.17) is also necessary for existence of a solution $u \in C^{2}(\bar{D})$.

Applying the method of continuity, and by results proved by Caffarelli, Nirenberg and Spruck in [6] and Krylov in [23], as well as by the iterration or $L^{p}$ theory given by Kohn and Nirenberg [21], it suffices to prove $C^{2}$ a priori estimates for solutions in Theorems 1.1-1.3. We will organized the rest of the paper as follows. In Section 2, we provide some preliminary results. In Section 3, we proved results of the subsolution. Finally, the main part of the paper, in Section 4, we provide $C^{2}$ a priori estimates.

2. Preliminary. From now on, we will always let $\Gamma$ be a symmetric convex cone in $\mathbb{R}^{n}$ with $\Gamma_{n} \subset \Gamma \subset \Gamma_{1}$, and $f(\lambda(H))=F(H)$ be an increasing, concave, nonnegative function on $\mathcal{M}(n, \Gamma)$. Let $\Gamma^{\prime}=\left\{\left(\lambda_{1}, \cdots, \lambda_{n-1}\right) \in \mathbb{R}^{n-1}: \lambda=\left(\lambda_{1}, \cdots, \lambda_{n}\right) \in\right.$ $\Gamma\}$ be the projection of $\Gamma$ into $\mathbb{R}^{n-1}$. It is easy to see that

$$
\Gamma_{n}^{\prime}=\Gamma_{n-1}\left(\mathbb{R}^{n-1}\right)=\left\{\left(\lambda_{1}, \cdots, \lambda_{n-1}\right): \lambda_{j}>0\right\}, \quad \Gamma_{1}^{\prime}=\mathbb{R}^{n-1} .
$$

For any $n \times n$ hermitian matrix $H=\left[h_{i \bar{j}}\right]$, we let $H^{\prime}=\left[h_{\alpha \bar{\beta}}\right]_{(n-1) \times(n-1)}$. Let $\lambda\left(H^{\prime}\right)$ be a vector formed by all eigenvalues of $H^{\prime}$. The following lemmas were proved in $[8]$.

Lemma 2.1. (Maximum Principle). Let $D$ be a bounded domain in $\mathbb{C}^{n}$ with $C^{1}$ boundary. Let $u, v \in C^{2}(D) \cap C(\bar{D})$ so that $H(u)(z) \in \mathcal{M}(n, \Gamma)$ for all $z \in D$. Assume that at every point $z \in D, \lambda(H(v(z)))$ lies outside the set $\Gamma(z, u)=\{\lambda \in \Gamma: f(\lambda)>$ $f(\lambda(H(u)(z))\}$. If $u \leq v$ on $\partial D$ then $u \leq v$ in $D$. 
LEMMA 2.2. With the notation above, we have

(2.2) $\lambda^{\prime}(H)=\left(\lambda_{1}(H), \cdots, \lambda_{n-1}(H)\right)=\lambda\left(H^{\prime}\right)+o(1), \quad \lambda_{n}(H)=h_{n \bar{n}}\left(1+O\left(1 / h_{n \bar{n}}\right)\right)$, as $h_{n \bar{n}} \rightarrow+\infty$.

LEMma 2.3. Let $\Gamma$ be a convex cone in $\mathbb{R}^{n}$ so that $\Gamma_{n} \subseteq \Gamma \subseteq \Gamma_{1}$. Let $\Gamma^{\prime}=$ $\left\{\left(\lambda_{1}, \cdots, \lambda_{n-1}\right): \lambda \in \Gamma\right\}$. If $\lambda(H) \in \Gamma$ then $\lambda\left(H^{\prime}\right) \in \Gamma^{\prime}$ where $H^{\prime}=\left(h_{\alpha \bar{\beta}}\right)_{(n-1) \times(n-1)}$.

Proof. Since $\lambda(H) \in \Gamma$, there is $\epsilon>0$ so that $\lambda\left(H-\epsilon I_{n}\right) \in \Gamma$. Let $E_{i j}$ be an $n \times n$ matrix with entries 1 at $(i, j)$ position, others are zero. Let $H(t)=H+t E_{n n}$. Then

$$
\lambda^{\prime}(H(t))=\lambda\left(H^{\prime}\right)+o(1) \in \Gamma^{\prime}, \quad \text { as } t \rightarrow \infty .
$$

Let $t \rightarrow+\infty$, we have $\lambda\left(H^{\prime}\right) \in \overline{\Gamma^{\prime}}$, and so $\lambda\left(H^{\prime}-\epsilon I_{n-1}\right) \in \overline{\Gamma^{\prime}}$. Thus $\lambda^{\prime}(H) \in \Gamma^{\prime}$.

The following proposition is proved by M. Marcus in [31] by using knowledge of probability, we will provide a direct proof here.

Proposition 2.4. Let $A=\left[a_{i \bar{j}}\right]$ and $B=\left[b_{i \bar{j}}\right]$ be hermitian matrices over $\mathbb{C}$. Let $\lambda_{1} \leq \cdots \leq \lambda_{n}$ be all eigenvalues of $A$ and let $\mu_{1} \geq \cdots \geq \mu_{n}$ be all eigenvalues of $B$. Then

$$
\operatorname{tr}(A B) \geq \sum_{k=1}^{n} \lambda_{k} \mu_{k}
$$

Proof. Without loss of generality, we may assume $A$ and $B$ are positive definite, otherwise, we will add them by $c I_{n}$ with large positive $c$. The proposition is true when $n=1$. Assume that it is true for $n$, we will prove that it is ture for $n+1$. Without loss of generality, we may assume that $A$ is a diagonal matrix, say, $A=$ $\operatorname{Diag}\left(\mu_{1}, \cdots, \mu_{n}, \mu_{n+1}\right)$. Since

$$
\operatorname{tr}(A B)=\operatorname{tr}\left(\left(A-\mu_{n+1} I_{n+1}\right) B\right)+\mu_{n+1} \operatorname{tr}(B) .
$$

Let $B^{\prime}=\left[b_{\alpha \bar{\beta}}\right]_{1 \leq \alpha, \beta \leq n}$, and let $\lambda_{1}^{\prime} \leq \lambda_{2}^{\prime} \cdots, \leq \lambda_{n}^{\prime}$ be the all eigenvalues of $B^{\prime}$. Let $\lambda_{1} \leq \lambda_{2} \leq \cdots \leq \lambda_{n} \leq \lambda_{n+1}$ be all eigenvalues of $B$. We claim that $\lambda_{j} \leq \lambda_{j}^{\prime}$ for all $1 \leq j \leq n$. Write

$$
B=\left[\begin{array}{cc}
B^{\prime} & b^{\prime} \\
\left(b^{\prime}\right)^{T} & b
\end{array}\right], \quad b^{\prime}=\left[\begin{array}{c}
b_{1} \\
\cdot \\
\cdot \\
\cdot \\
b_{n-1}
\end{array}\right]
$$

Hence

$$
g_{n+1}(\lambda)=\operatorname{det}\left(\lambda I_{n+1}-B\right)=\prod_{j=1}^{n}\left(\lambda-\lambda_{j}^{\prime}\right)\left(\lambda-b-\frac{1}{\lambda-\lambda_{j}^{\prime}} \sum_{k=1}^{n}\left|b_{k}\right|^{2}\right)
$$

we have

$$
g_{n+1}\left(\lambda_{\ell}^{\prime}\right)=-\prod_{j \neq \ell}^{n}\left(\lambda_{\ell}^{\prime}-\lambda_{j}^{\prime}\right) \sum_{k=1}^{n}\left|b_{k}\right|^{2}=(-1)^{n+1-\ell} A_{\ell}, \quad A_{\ell} \geq 0 .
$$


If $\sum_{k=1}^{n}\left|b_{k}\right|^{2}=0$ then it is easy. We may assume that $\sum_{k=1}^{n}\left|b_{k}\right|^{2}>0$ or $A_{\ell}>0$ for all $\ell$. We will continue our discussion in the following two cases.

Case 1. If $n+1$ is even then $(-1)^{\ell} g_{n+1}\left(\lambda_{\ell}^{\prime}\right) \geq 0$, then $g_{n+1}(\lambda)$ has zeros in $\left(\lambda_{j}^{\prime}, \lambda_{j+1}^{\prime}\right]$ for all $j=1, \cdots, n-1$. Since $g_{n+1}(\lambda) \rightarrow+\infty$ as $|\lambda| \rightarrow+\infty$, this implies that $g_{n+1}(\lambda)$ has a zero $\lambda_{1} \leq \lambda_{1}^{\prime}$ and a zero $\lambda_{n+1}>\lambda_{n}^{\prime}$. So $\lambda_{j} \leq \lambda_{j}^{\prime}$ for all $j=1, \cdots, n$.

Case 2. If $n+1$ is odd then $(-1)^{\ell} g_{n+1}\left(\lambda_{\ell}^{\prime}\right) \leq 0$. Since $g_{n+1}(\lambda) \rightarrow-\infty$ as $\lambda \rightarrow-\infty$, with similar argument above, we have $g_{n+1}(\lambda)$ has zeros $\lambda_{j}$ with $\lambda_{j}^{\prime} \geq \lambda_{j}$ for $j=$ $1,2, \cdots, n$. So the proof of the claim is complete. Now since

$$
\operatorname{tr}\left(\left(A-\mu_{n+1} I_{n+1}\right) B\right)=\sum_{k=1}^{n}\left(\mu_{k}-\mu_{n+1}\right) b_{k \bar{k}} \geq \sum_{k=1}^{n}\left(\mu_{k}-\mu_{n+1}\right) \lambda_{k}^{\prime}
$$

Therefore

$$
\begin{aligned}
\operatorname{tr}(A B) & \geq \sum_{k=1}^{n}\left(\mu_{k}-\mu_{n+1}\right) \lambda_{k}^{\prime}+\mu_{n+1} \operatorname{tr}(B) \\
& =\sum_{k=1}^{n} \mu_{k} \lambda_{k}^{\prime}+\mu_{n+1} \lambda_{n+1}+\mu_{n+1} \sum_{k=1}^{n}\left(\lambda_{k}-\lambda_{k}^{\prime}\right) \\
& =\sum_{k=1}^{n+1} \mu_{k} \lambda_{k}+\sum_{k=1}^{n} \mu_{k}\left(\lambda_{k}^{\prime}-\lambda_{k}\right)+\mu_{n+1} \sum_{k=1}^{n}\left(\lambda_{k}-\lambda_{k}^{\prime}\right) \\
& =\sum_{k=1}^{n+1} \mu_{k} \lambda_{k}+\sum_{k=1}^{n}\left(\mu_{k}-\mu_{n+1}\right)\left(\lambda_{k}^{\prime}-\lambda_{k}\right) \\
& \geq \sum_{k=1}^{n+1} \mu_{k} \lambda_{k},
\end{aligned}
$$

and the proof of the proposition is complete by mathematics induction.

Next we gives a simple version of continous case of the previous proposition.

Proposition 2.5. Let $f(t)$ be decreasing on $[0,1]$ and $g(t)$ is increasing on $[0,1]$. Let $\phi(x, y) \geq 0$ be a measurable function on $[0,1] \times[0,1]$ so that $\phi(x, y)=\phi(y, x)$ and

$$
\int_{0}^{1} \phi(x, y) d y=1, \quad \text { a.e. } \quad x \in[0,1] .
$$

Then

$$
\int_{0}^{1} \int_{0}^{1} \phi(x, y) f(x) g(y) d x d y \geq \int_{0}^{1} f(x) g(x) d x
$$

Proof. For any $x, y \in[0,1]$, since $(f(x)-f(y))(g(x)-g(y)) \leq 0$, we have

$$
f(x) g(x)+f(y) g(y)-f(x) g(y)-f(y) g(x) \leq 0 .
$$

Therefore

$$
f(x) g(x)+f(y) g(y) \leq f(x) g(y)+f(y) g(x)
$$


and

$$
\begin{aligned}
& \int_{0}^{1} \int_{0}^{1} \phi(x, y)(f(y) g(x)+f(x) g(y)) d x d y \\
& \geq \int_{0}^{1} \int_{0}^{1} \phi(x, y)[f(x) g(x)+f(y) g(y)] d y \\
& =2 \int_{0}^{1} f(x) g(x) d x
\end{aligned}
$$

Since

$$
\int_{0}^{1} \int_{0}^{1} \phi(x, y) f(y) g(x) d x d y=\int_{0}^{1} \int_{0}^{1} \phi(y, x) f(x) g(y) d x d y
$$

we have

$$
\begin{aligned}
& \int_{0}^{1} \int_{0}^{1} \phi(x, y)(f(y) g(x)+f(x) g(y)) d x d y \\
& \left.=\int_{0}^{1} \int_{0}^{1} \phi(y, x) f(x) g(y)+\int_{0}^{1} \int_{0}^{1} \phi(x, y) f(x) g(y)\right) d x d y \\
& =\int_{0}^{1} \int_{0}^{1}(\phi(y, x)+\phi(x, y)) f(x) g(y) d x d y
\end{aligned}
$$

Therefore

$$
\begin{aligned}
\int_{0}^{1} \int_{0}^{1} \phi(x, y) f(x) g(y) d x d y & =\int_{0}^{1} \int_{0}^{1} \frac{\phi(y, x)+\phi(x, y)}{2} f(x) g(y) d x d y \\
& \geq \int_{0}^{1} f(x) g(x) d x
\end{aligned}
$$

and the proof of the lemma is complete.

3. Existence of subsolutions. We first prove the following theorem on defining function.

Theorem 3.1. Let $\Gamma$ be a convex cone in $\mathbb{R}^{n}$ with $\Gamma_{n} \subset \Gamma \subset \Gamma_{1}$. Let $\rho \in C^{\infty}(\bar{D})$ be a defining function for $D$ so that $\lambda(H(\rho)(z)) \in \Gamma$ for all $z \in \partial D$. Then there is a defining function $\rho^{0} \in C^{\infty}(\bar{D})$ for $D$ so that $\lambda\left(H\left(\rho^{0}\right)\right) \in \Gamma$ on $\bar{D}$.

Proof. Since $\Gamma$ is an open set, and $\lambda(H(\rho)(z)) \in \Gamma$ for all $z \in \partial D$, and $\partial D$ is compact. By continuity, there is a $\delta>0$ so that $\lambda(H(\rho)(z)) \in \Gamma$ for all $\{z \in \bar{D}$ : $\rho(z) \geq-\delta\}$. Now we choose a convex increasing function $g(t) \in C^{\infty}(-\infty, 0]$ so that $g(0)=0, g(t)=-1$ when $t \leq-\delta$ and $g^{\prime}(t)>0$ for $t \in(-\delta, 0)$. Let

$$
\rho^{1}(z)=g(\rho(z))
$$

It is easy to see that $\rho^{1} \in C^{\infty}(\bar{D})$ and is a defining function for $D$. Since

$$
H\left(\rho^{1}\right)(z)=g^{\prime}(\rho) H(\rho)+g^{\prime \prime}(\rho) \partial \rho \otimes \bar{\partial} \rho(z)
$$

since $g^{\prime \prime} \geq 0, \partial \rho \otimes \bar{\partial} \rho$ is positive semi-definite, and $g^{\prime} \geq 0$, we have that $\lambda\left(H\left(\rho^{1}\right)(z)\right) \in$ $\bar{\Gamma}$ for all $z \in \bar{D}$ and $\lambda\left(H\left(\rho^{1}\right) \in \Gamma\right.$ for $\rho>-\delta$. We let $h \in C_{0}^{\infty}(D)$ so that $h(z) \geq 0$ on $D$ and $h(z)=1$ when $\rho(z)<-\delta / 2$. Let

$$
\rho^{0}(z)=\operatorname{ch}(z)|z|^{2}+\rho^{1}(z), \quad c>0
$$


It is obvious that $\rho^{0}$ is a defining function for $D$, and $\rho^{0}<0$ if $c>0$ is small enough. Moreover,

$H\left(\rho^{0}\right)=g^{\prime}(\rho) H(\rho)+g^{\prime \prime}(\rho) \partial \rho \otimes \bar{\partial} \rho(z)+\operatorname{ch}(z) I_{n}+c \partial h(z) \otimes z+c \bar{z} \otimes \bar{\partial} h(z)+c|z|^{2} H(h)$

It is easy to see that $\lambda\left(H\left(\rho^{0}\right)(z)\right) \in \Gamma$ when $\rho(z)<-\delta / 2$ for any $c>0$ since $h \equiv 1$ there. Now since $\lambda\left(H\left(\rho^{1}\right)\right) \in \Gamma$ for $\rho(z) \geq-3 \delta / 4$, those $\lambda$ contained in a compact subset of $\Gamma$, there is a positive distance from it to the boundary $\partial \Gamma$. Therefore, there is $c>0$ very small so that $\lambda\left(H\left(\rho^{0}\right)\right)=\lambda\left(H\left(\rho^{1}\right)+c H\left(h|z|^{2}\right)\right) \in \Gamma$. Thus, the proof of the theorem is complete.

TheOREM 3.2. Let $\Gamma$ be a convex symmetric cone in $\mathbb{R}^{n}$. Let $F$ be a concave increasing function on $\mathcal{M}(n, \Gamma)$ satisfying (1.7)-(1.11). Let $D$ be a smoothly bounded domain in $\mathbb{C}^{n}$ so that there is a defining function $\rho \in C^{\infty}(\bar{D})$ for $D$ with $H(\rho) \in$ $\mathcal{M}(n, \Gamma)$ and $F(H(\rho))>0$ on $\bar{D}$. If $\phi \in C^{\infty}(\partial D)$ and $\psi \in C^{\infty}(\bar{D})$ is positive, then there is $\underline{u} \in C^{\infty}(\bar{D})$ so that

$$
F(H(\underline{u})) \geq \psi(z)+1, \quad \text { in } D, \quad \text { and } \underline{u}=\phi \quad \text { on } \partial D .
$$

Proof. Let $\phi \in C^{\infty}(\partial D)$, we still use $\phi$ to denote its harmonic extension to $\bar{D}$. Let $C_{1}>0$ so that $\phi_{0}=\phi(z)+C_{1} \rho(z)$ satisfying $\lambda\left(H\left(\phi_{0}\right)(z)\right) \in \Gamma$ by assumption $H(\rho) \in \mathcal{M}(n, \Gamma)$ and (1.11). Let

$$
\underline{u}=\phi_{0}(z)+C \rho(z) .
$$

Then

$$
H(\underline{u})=H\left(\phi_{0}(z)\right)+C H(\rho(z)) .
$$

Since $F$ is concave on $\mathcal{M}(n, \Gamma)$ or $f$ is concave on $\Gamma$, we have

$$
\begin{aligned}
F(H(\underline{u})) & \geq \frac{F\left(2 H\left(\phi_{0}\right)\right)}{2}+\frac{F(2 C H(\rho))}{2} \\
& =\frac{F\left(2 H\left(\phi_{0}\right)\right)}{2}+\frac{f(2 C \lambda(H(\rho)))}{2} \\
& \geq \frac{f(2 C \lambda(H(\rho)))}{2} .
\end{aligned}
$$

By (1.11), for any compact subset $K$ and a positive constant $C_{0}$, there is $R=$ $R\left(K, C_{0}\right)>0$ so that

$$
f(R \lambda) \geq C_{0}, \quad \text { for all } \lambda \in K
$$

Now let $K=\{\lambda(H(\rho(z))): z \in \bar{D}\}$ is a compact set in $\Gamma$. Let

$$
C_{0}=2\left(\psi_{1}+1\right), \quad \psi_{1}=\max \{\psi(z): z \in \bar{D}\}
$$

and $2 C=R=R\left(C_{0}, K\right)$. Then we have

$$
F(H(\underline{u})(z)) \geq \psi_{1}+1 \quad \text { for } z \in \bar{D},
$$

and the proof is complete.

Finally, in this section, we shall prove the following proposition. 
Proposition 3.3. Let $D$ be a bounded domain in $\mathbb{C}^{n}$ with $\partial D$ is connected and $C^{\infty}$. If there is a defining function $\rho$ for $D$ so that $\lambda\left(\mathcal{L}_{\rho}\right) \in \Gamma_{k-1}$ on $\partial D$ then there is a defining function $\rho_{0}$ for $D$ so that $\lambda\left(H\left(\rho_{0}\right) \in \Gamma_{k}\right.$ on $\partial D$. Here $\mathcal{L}_{\rho}$ is the Levi-form of $\rho$ on $\partial D$.

Proof. Without loss of generality, we may assume that $|\partial \rho|^{2}=1 / 2$ on $\partial D$. Let

$$
\rho_{0}(z)=\rho(z)+C \rho(z)^{2}, \quad z \in D
$$

Then

$$
\mathcal{L}_{\rho}(z)=\mathcal{L}_{\rho_{0}}(z), \quad z \in \partial D
$$

and

$$
\lambda\left(H\left(\rho_{0}\right)(z)\right)=\left(\lambda^{\prime}(z), \lambda_{n}\right)=\left(\lambda\left(\mathcal{L}_{\rho}(z)\right)+o(1), C+O(1)\right), \quad \text { as } \quad C \rightarrow+\infty .
$$

Since $\lambda\left(\mathcal{L}_{\rho}(z)\right) \in \Gamma_{k-1}$ on $\partial D$ there is $a>0$ and $C>>1$ so that

$$
\sigma^{(j)}\left(\lambda^{\prime}(z)+o(1)\right) \geq a, \quad z \in \partial D, \quad \text { and } 1 \leq j \leq k-1 .
$$

This imples that when $C>1$ is large enough we have $\sigma^{(j)}(\lambda(H(\rho))>0$ on $\partial D$ for all $1 \leq j \leq k$, and so $\lambda(H(\rho)) \in \Gamma_{k}$. Therefore, the proof is complete.

4. A priori estimates up to the second derivatives. Let $D$ be a bounded domain in $\mathbb{C}^{n}$ with smooth boundary. Let $u$ be a real-valued function on $D$. We consider the Dirichlet boundary value problem:

$$
F(H(u))=f(\lambda(H(u)))=\psi(z), \quad z \in D ; \quad u=\phi \quad \text { on } \partial D
$$

with the assumptions: $\psi \in C^{\infty}(\bar{D})$ and $\psi(z)>0$ on $\bar{D}$. Moreover, we assume that $\phi \in C^{\infty}(\partial D)$ has an extension $\underline{u}$ on $\bar{D}$ so that

$$
\lambda(H(\underline{u})(z)) \in \Gamma \quad \text { and } F(H(\underline{u})(z)) \geq \psi(z)+\epsilon, \quad z \in D
$$

for some $\epsilon>0$. We say that $u \in C^{2}(D)$ is an admissible solution of (4.1) if $\lambda(H(u)) \epsilon$ $\Gamma$ on $D$. Then we shall prove the following lemma.

LEMMA 4.1. Let $\phi \in C^{\infty}(\partial D)$ have an extension $\underline{u}$ satisfying (4.2). Let (4.1) have an admissible solution $u \in C^{1}(\bar{D})$. Then

$$
\|u\|_{C^{1}(\bar{D})} \leq C_{1} .
$$

where $C_{1}$ is a constant depending only on $\|\phi\|_{C(\partial D)},\|\underline{u}\|_{C^{1}(\bar{D})},\|\psi\|_{C^{1}(\bar{D})}$ and $\epsilon$.

Proof. We still use $\phi$ as its harmonic extension of $\phi$ from $\partial D$ to $\bar{D}$. Then $u-\phi=0$ on $\partial D$ and $\Delta(u-\phi)=\Delta u>0($ since $\Delta u>0)$. By maximum principle, we have that

$$
u(z)-\phi(z) \leq 0, \quad z \in \bar{D}
$$

Moreover,

$$
D_{\nu}(u-\phi)(z) \geq 0, \quad z \in \partial D
$$

where $\nu$ is the outer unit normal vector to $\partial D$. 
Since $f(\lambda(H(\underline{u}))) \geq f(\lambda(H(u))$ and $\underline{u}=u$ on $\partial D$ (of course, $\underline{u} \leq u$ on $\partial D$ ), by the Maximum Principle or Lemma 2.1, we have $\underline{u} \leq u$ on $\bar{D}$. Since $\underline{u}-u \leq 0$ on $D$ and $\underline{u}-u=0$ on $\partial D$, this implies that

$$
D_{\nu}(\underline{u}-u) \geq 0, \quad \text { on } \partial D
$$

Therefore,

$$
\|u\|_{C(\bar{D})} \leq\|\phi\|_{C(\bar{D})}+\|\underline{u}\|_{C(\bar{D})} \leq C\left(\|\underline{u}\|_{C(\bar{D})},\|\phi\|_{C(\partial D)}\right)
$$

where $C$ is a constant depending only on $\|\phi\|_{C(\partial D)}$ and $\|\underline{u}\|_{C(\bar{D})}$ and $\partial D$. Moreover,

$$
-\|\phi\|_{C^{1}(\partial D)} \leq D_{\nu} \phi(z) \leq D_{\nu} u(z) \leq D_{\nu} \underline{u}(z) \leq\|\underline{u}\|_{C^{1}(\bar{D})}, \quad z \in \partial D
$$

Since $u=\phi$ on $\partial D$, we have

$$
|\nabla u(z)| \leq\|\phi\|_{C^{1}(\partial D)}+\|\underline{u}\|_{C^{1}(\bar{D})}, \quad z \in \partial D .
$$

In order to estimate $|\nabla u|$ on $D$, we let

$$
F^{i \bar{j}}=\frac{\partial F}{\partial u_{i \bar{j}}}(H(u)), \quad 1 \leq i, j \leq n .
$$

Then $L=F^{i \bar{j}} \partial_{i \bar{j}}$ is an elliptic operator. Since $F$ is concave function on $\mathcal{M}(n, \Gamma)$ and since (4.1) and (4.2), we have

$$
\epsilon \leq F(H(\underline{u}))-F(H(u)) \leq F^{i \bar{j}}(H(u))\left(\underline{u}_{i \bar{j}}-u_{i \bar{j}}\right)=L(\underline{u}-u), \quad \text { on } D .
$$

$F(H(u)(z))=\psi(z)$ on $D$ and the Chain Rules imply that

$$
\frac{\partial \psi(z)}{\partial z_{i}}=\frac{\partial F(H(u)(z))}{\partial z_{i}}=F^{k \bar{\ell}}(H(u)) \frac{\partial u_{k \bar{\ell}}}{\partial z_{i}}=L \partial_{i} u
$$

Similarly, we have with $\psi_{i}=\frac{\partial \psi}{\partial z_{i}}$ and $\psi_{\bar{j}}=\frac{\partial \psi}{\partial \bar{z}_{j}}$,

$$
L u_{i}=\psi_{i}, \quad L u_{\bar{j}}=\psi_{\bar{j}}, \quad L \frac{\partial u}{\partial x_{j}}=\frac{\partial \psi}{\partial x_{j}}, \quad L \frac{\partial u}{\partial y_{j}}=\frac{\partial \psi}{\partial y_{j}}, \quad 1 \leq i, j \leq n .
$$

Therefore, by (4.11) and (4.12)

$$
L\left(a \frac{\partial u}{\partial x_{j}}+b \frac{\partial u}{\partial y_{j}}+C(\underline{u}-u)\right)=a \frac{\partial \psi}{\partial x_{j}}+b \frac{\partial \psi}{\partial y_{j}}+C L(\underline{u}-u) \geq a \frac{\partial \psi}{\partial x_{j}}+b \frac{\partial \psi}{\partial y_{j}}+C \epsilon \geq 0
$$

where $C=\frac{2}{\epsilon}\|\nabla \psi\|_{C(\bar{D})}$ and $a^{2}+b^{2}=1$. Thus, $a \frac{\partial u}{\partial x_{j}}+b \frac{\partial u}{\partial y_{j}}+C(\underline{u}-u)$ attains its maximum over $\bar{D}$ at some point on $\partial D$. Therefore, by taking $a= \pm 1, b=0$ and $a=0, b= \pm 1$, we have

$$
|\nabla u(z)| \leq 2 n C\|\underline{u}-u\|_{C(\bar{D})}+\|\nabla u\|_{C(\partial D)}, \quad z \in \bar{D} .
$$

Therefore,

$$
\|u\|_{C^{1}(\bar{D})} \leq C\left(\|\phi\|_{C(\partial D)},\|\underline{u}\|_{C^{1}(\bar{D})},\|\psi\|_{C^{1}(\bar{D})}, \epsilon\right) .
$$


where $C\left(\|\phi\|_{C(\partial D)},\|\underline{u}\|_{C^{1}(\bar{D})},\|\psi\|_{C^{1}(\bar{D})}, \epsilon\right)$ is a constant depending only on $\|\phi\|_{C^{1}(\partial D)},\|\underline{u}\|_{C^{1}(\bar{D})},\|\psi\|_{C^{1}(\bar{D})}$ and $\epsilon$. Therefore, the proof of the lemma is complete.

Lemma 4.2. With the assumption of Lemma 4.1, if $u \in C^{2}(\bar{D})$ is an admissible solution of (4.1), then

$$
\|u\|_{C^{2}(\bar{D})} \leq C\left(\|u\|_{C^{1}(\bar{D})},\|\psi\|_{C^{2}(\bar{D})}, \epsilon,\|u\|_{C^{2}(\partial D)}\right)
$$

where $C$ is a constant depending only on $\|u\|_{C^{1}(\bar{D})},\|\psi\|_{C^{2}(\bar{D})}, \epsilon$ and $\|u\|_{C^{2}(\partial D)}$.

Proof. For any $\xi \in S^{2 n-1}$, we consider

$$
U(z)=D_{\xi \xi} u(z)+C(\underline{u}-u), \quad D_{\xi \xi}=D_{\xi}^{2}, \quad D_{\xi}=\sum_{j=1}^{n} \xi_{n+j} \frac{\partial u}{\partial x_{j}}+\sum_{j=1}^{n} \xi_{j} \frac{\partial u}{\partial y_{j}}
$$

Let

$$
F^{i \bar{j}, k \bar{\ell}}=\frac{\partial^{2} F}{\partial u_{i \bar{j}} \partial u_{k \bar{\ell}}} .
$$

Then, by the concavity of $F$, we have $F^{i \bar{j}, k \bar{\ell}} a_{i \bar{j}} a_{k \bar{\ell}} \leq 0$ for any hermitian matrix $A=\left[a_{i \bar{j}}\right]$. Therefore,

$$
\begin{aligned}
D_{\xi} D_{\xi} \psi(z) & =D_{\xi} D_{\xi} F(H(u)) \\
& =D_{\xi}\left(F^{i \bar{j}} D_{\xi} u_{i \bar{j}}\right) \\
& =F^{i \bar{j} k \bar{\ell}} D_{\xi} u_{k \bar{\ell}} D_{\xi} u_{i \bar{j}}+F^{i \bar{j}} D_{\xi}^{2} u_{i \bar{j}} \\
& =F^{i \bar{j} k \bar{\ell}} D_{\xi} u_{k \bar{\ell}} D_{\xi} u_{i \bar{j}}+F^{i \bar{j}} \frac{\partial^{2}}{\partial z_{i} \partial \bar{z}_{j}} D_{\xi \xi} u \\
& \leq L D_{\xi \xi} u .
\end{aligned}
$$

If we let $C=\frac{1}{\epsilon}\left\|D^{2} \psi\right\|_{C(\bar{D})}$ then

$$
L\left(D_{\xi \xi} u+C(\underline{u}-u)\right) \geq D_{\xi \xi} \psi(z)+C \epsilon \geq 0 .
$$

Therefore $D_{\xi \xi} u+C(\underline{u}-u)$ attains its maximum over $\bar{D}$ at some point $z_{0} \in \partial D$. Thus

$$
D_{\xi \xi} u(z) \leq C\left(\|\phi\|_{C^{2}(\partial D)},\|\psi\|_{C^{2}(\bar{D})}, \epsilon,\|\underline{u}\|_{C^{1}(\bar{D})}\right)+\left\|D^{2} u\right\|_{C(\partial D)}=C_{2}
$$

Since

$$
\Delta u(z)>0, \quad z \in D
$$

we have

$$
C_{2} \geq u_{x_{j} x_{j}} \geq-(2 n-1) C_{2}, \quad C_{2} \geq u_{y_{j} y_{j}} \geq-(2 n-1) C_{2} .
$$

Thus

$$
\left|u_{x_{j} x_{j}}\right|, \quad\left|u_{y_{j}, y_{j}}\right| \leq(2 n-1) C_{2}, \quad 1 \leq j \leq n .
$$


Since

$$
\pm 2 D_{x_{i}} D_{x_{j}} u=D_{x_{i} \pm x_{j}}^{2} u(z)-\left(D_{x_{i}}^{2} u+D_{x_{j}}^{2} u\right) \leq(4 n-1) C_{2}
$$

and

$$
\pm 2 D_{x_{i}} D_{y_{j}} u=D_{x_{i} \pm y_{j}}^{2} u(z)-\left(D_{x_{i}}^{2} u+D_{y_{j}}^{2} u\right) \leq(4 n-1) C_{2}
$$

and

$$
\pm 2 D_{y_{i}} D_{y_{j}} u=D_{y_{i} \pm y_{j}}^{2} u(z)-\left(D_{y_{i}}^{2} u+D_{y_{j}}^{2} u\right) \leq(4 n-1) C_{2} .
$$

We have

$$
\left|D_{x_{i}} D_{x_{j}} u\right|+\left|D_{y_{i}} D_{y_{j}} u\right|+\left|D_{x_{i}} D_{y_{j}} u\right| \leq 6 n C_{2} .
$$

Thus

$$
\|u\|_{C^{2}(\bar{D})} \leq 6 n^{2} C_{2}
$$

and the proof of the lemma is complete.

To complete the estimates for second derivatives of $u$, it suffices to estimate $\left|\nabla^{2} u\right|$ on $\partial D$ (or $\left.C_{2}\right)$. Since $u=\phi \in C^{\infty}(\partial D)$, we have

$$
\left|D_{T}^{2} u(z)\right| \leq C\left(\|\phi\|_{C^{2}(\partial D)},\|u\|_{C^{1}(\bar{D})}\right), \quad z \in \partial D
$$

for any smooth unit tangent vector $T$ to $\partial D$ at $z \in \partial D$. What we need to estimate are $\left|D_{T} D_{\nu} u\right|$ on $\partial D$ and $\left|D_{\nu}^{2} u\right|$ on $\partial D$. Which will be given by the following two lemmas.

LEMma 4.3. Let $\rho$ be a defining function for $D$ with $D_{\nu} \rho=1$ on $\partial D$. With the assumptions of Lemma 4.1, we have

$$
\left|D_{T} D_{\nu} u(z)\right| \leq C\left(\|\phi\|_{C^{3}(\partial D)},\|\psi\|_{C^{2}(\bar{D})}, \epsilon\right), \quad z \in \partial D .
$$

Proof. Let $z_{0} \in \partial D$ be an arbitrary point. Without loss of generality, by shift and rotation, we may assume that $z_{0}=0, \frac{\partial \rho}{\partial x_{j}}(0)=0$ for $1 \leq j \leq n-1$ and $\frac{\partial \rho}{\partial y_{j}}(0)=0$ for all $1 \leq j \leq n$. Moreover, nearby $z_{0}=0$, we can write

$$
\rho(z)=-x_{n}+\operatorname{Re} \sum_{i, j=1}^{n} \rho_{i j}(0) z_{i} z_{j}+\sum_{i, j=1}^{n} \rho_{i \bar{j}}(0) z_{i} \bar{z}_{j}+P(z)+R(z)
$$

where $P(z)$ is cubic polynomial in $z$ and $\bar{z}$, and $|R(z)| \leq C|z|^{4}$.

Let $t_{\alpha}=y_{\alpha}$ if $1 \leq \alpha \leq n$ and $t_{\alpha+n}=x_{\alpha}$ if $1 \leq \alpha \leq n-1$. Let

$$
a_{\alpha}(z)=-\frac{\partial \rho}{\partial t_{\alpha}} / \frac{\partial \rho}{\partial x_{n}}, \quad 1 \leq \alpha \leq 2 n-1
$$

Then

$$
a_{\alpha}(0)=0, \quad T=\frac{\partial}{\partial t_{\alpha}}+a_{\alpha} \frac{\partial}{\partial x_{n}}
$$


is a tangent vector to $\partial D$ near $z=0 \in \partial D$. We write

$$
a_{\alpha}(z)=\sum_{\beta=1}^{2 n-1} b_{\alpha \beta} t_{\beta}+b_{\alpha} x_{n}+O\left(|t|^{2}+x_{n}^{2}\right), \quad z \in \bar{D} \text { near } 0
$$

Let

$$
T_{\alpha}=\frac{\partial}{\partial t_{\alpha}}+\sum_{\beta=1}^{2 n-1} b_{\alpha \beta} t_{\beta} \frac{\partial}{\partial x_{n}}, \quad b_{\alpha \beta}=\frac{\partial a_{\alpha}}{\partial t_{\beta}}(0) .
$$

Then $T=T_{\alpha}+b_{\alpha} x_{n} \frac{\partial}{\partial x_{n}}+O\left(|z|^{2}\right) \frac{\partial}{\partial x_{n}}$ and

$$
T_{\alpha}(u-\bar{u})=O\left(|t|^{2}\right), \quad \text { on } \partial D .
$$

Since

$$
\partial_{i} t_{\beta}= \begin{cases}-\frac{\sqrt{-1}}{2} \delta_{i \beta} & \text { if } 1 \leq \beta \leq n \\ \frac{1}{2} \delta_{i \beta-n}, & \text { if } \beta>n\end{cases}
$$

and

$$
\partial_{\bar{j}} t_{\beta}= \begin{cases}\frac{\sqrt{-1}}{2} \delta_{j \beta} & \text { if } 1 \leq \beta \leq n-1 \\ \frac{1}{2} \delta_{j \beta-n}, & \text { if } \beta>n\end{cases}
$$

Therefore,

$$
\begin{aligned}
L T_{\alpha} u= & T_{\alpha} \psi(z)+\sum_{\beta} b_{\alpha \beta} F^{i \bar{j}}\left(\partial_{i} t_{\beta} \partial_{\bar{j}} u_{x_{n}}+\partial_{\bar{j}} t_{\beta} \partial_{i} u_{x_{n}}\right) \\
= & T_{\alpha} \psi(z)+2 \sum_{\beta<2 n} b_{\alpha \beta} F^{i \bar{j}}\left(\partial_{i} t_{\beta} \partial_{\bar{j}} u_{n}+\partial_{\bar{j}} t_{\beta} \partial_{i} u_{\bar{n}}\right) \\
& +2 \sum_{\beta} b_{\alpha \beta} F^{i \bar{j}}\left(\sqrt{-1} \partial_{i} t_{\beta} \partial_{\bar{j}} u_{y_{n}}-\sqrt{-1} \partial_{\bar{j}} t_{\beta} \partial_{i} u_{y_{n}}\right) \\
= & T_{\alpha} \psi(z)+\sqrt{-1} \sum_{\beta=1}^{n} b_{\alpha \beta} F^{i \bar{j}}\left(-\delta_{i \beta} u_{n \bar{j}}+\delta_{j \beta} u_{i \bar{n}}\right) \\
& +\sum_{\beta=1}^{n-1} b_{\alpha \beta+n} F^{i \bar{j}}\left(\delta_{i \beta} u_{n \bar{j}}+\delta_{j \beta} u_{i \bar{n}}\right)+2 \sqrt{-1} \sum_{\beta} b_{\alpha \beta} F^{i \bar{j}}\left(\partial_{i} t_{\beta} \partial_{\bar{j}} u_{y_{n}}-\partial_{\bar{j}} t_{\beta} \partial_{i} u_{y_{n}}\right) \\
= & T_{\alpha} \psi(z)+2 \sqrt{-1} \sum_{\beta=1}^{n} b_{\alpha \beta} \operatorname{Im}\left(F^{i \bar{\beta}} u_{i \bar{n}}\right)+2 \sum_{\beta=1}^{n-1} b_{\alpha \beta+n} \operatorname{Re}\left(F^{\beta \bar{j}} u_{n \bar{j}}\right) \\
& +2 \sqrt{-1} \sum_{\beta} b_{\alpha \beta} F^{i \bar{j}}\left(\partial_{i} t_{\beta} \partial_{\bar{j}} u_{y_{n}}-\partial_{\bar{j}} t_{\beta} \partial_{i} u_{y_{n}}\right)
\end{aligned}
$$

Since $b_{\alpha \beta}$ are real number, we have

$2 \sqrt{-1} \sum_{i=1}^{n} \sum_{\beta=1}^{n} b_{\alpha \beta} \operatorname{Im}\left(F^{i \bar{\beta}} u_{i \bar{n}}\right)+2 \sqrt{-1} \operatorname{Re} \sum_{\beta} b_{\alpha \beta} F^{i \bar{j}}\left(\partial_{i} t_{\beta} \partial_{\bar{j}} u_{y_{n}}-\partial_{\bar{j}} t_{\beta} \partial_{i} u_{y_{n}}\right)=0$ 
By rotation of first $(n-1)$ variables, we may assume that

$$
F^{\beta \bar{j}}=\delta_{j \beta} F^{\beta \bar{\beta}}, \quad \operatorname{Re} F^{\beta \bar{n}}=\operatorname{Re} u_{j \bar{n}}=0, \quad 1 \leq \beta, j \leq n-1 .
$$

Thus

$$
\begin{aligned}
2 \sum_{\beta=1}^{n-1} b_{\alpha \beta+n} \operatorname{Re}\left(F^{\beta \bar{j}} u_{n \bar{j}}\right) & =2 \sum_{j, \beta=1}^{n-1} b_{\alpha \beta+n} \operatorname{Re}\left(F^{\beta \bar{j}} u_{n \bar{j}}\right)+2 \sum_{\beta=1}^{n-1} b_{\alpha \beta+n} \operatorname{Re}\left(F^{\beta \bar{n}} u_{n \bar{n}}\right) \\
& =2 \sum_{\beta=1}^{n-1} b_{\alpha \beta+n} \operatorname{Re}\left(F^{\beta \bar{\beta}} u_{n \bar{\beta}}\right)+2 \sum_{\beta=1}^{n-1} b_{\alpha \beta+n} u_{n \bar{n}} \operatorname{Re}\left(F^{\beta \bar{n}}\right) \\
& =2 \sum_{\beta=1}^{n-1} b_{\alpha \beta+n} F^{\beta \bar{\beta}} \operatorname{Re}\left(u_{n \bar{\beta}}\right) \\
& =0 .
\end{aligned}
$$

Since

$-2 \operatorname{Im} \sum_{\beta} b_{\alpha \beta} F^{i \bar{j}}\left(\partial_{i} t_{\beta} \partial_{\bar{j}} u_{y_{n}}-\partial_{\bar{j}} t_{\beta} \partial_{i} u_{y_{n}}\right) \geq-C \operatorname{tr}\left(F^{i \bar{j}}\right)-\frac{1}{4} F^{i \bar{j}} \partial_{i}\left(u_{t_{\beta}}-\underline{u}_{t_{\beta}}\right) \partial_{\bar{j}}\left(u_{t_{\beta}}-\underline{u}_{t_{\beta}}\right)$.

Therefore

$$
\pm L T_{\alpha}(u-\underline{u}) \geq-C\left(\operatorname{tr}\left(F^{i \bar{j}}\right)+1\right)-F^{i \bar{j}} \partial_{i}\left(u_{t_{\beta}}-\underline{u}_{t_{\beta}}\right) \partial_{\bar{j}}\left(u_{t_{\beta}}-\underline{u}_{t_{\beta}}\right) .
$$

Since $\lambda\left(H(\underline{u}) \in \Gamma\right.$ and $f\left(\lambda(H(\underline{u})) \geq \psi+\epsilon\right.$, there is $\epsilon_{1}>0$ such that $\lambda(H(\underline{u})-$ $\left.\left.2 \epsilon_{1} I_{n}\right)\right) \in \Gamma$ for all $z \in \bar{D}$, and $f\left(\lambda\left(H(\underline{u})-2 \epsilon_{1} I_{n}\right)\right) \geq \psi(z)+\epsilon_{0}\left(\epsilon_{1}\right)>0$ on $\bar{D}$, where $\epsilon_{0}\left(\epsilon_{1}\right) \leq \epsilon$ is positive constant depending only on $f \epsilon$ and sufficiently small $\epsilon_{1}$. Now we let

$$
V^{ \pm}(z)= \pm T_{\alpha}(u-\underline{u})+\sum_{\beta=1}^{2 n-1}\left(u_{t_{\beta}}-\underline{u}_{t_{\beta}}\right)^{2}+A\left(\underline{u}-u-\epsilon_{1}|z|^{2}\right)
$$

Then

$$
\begin{aligned}
& L\left(A\left(\underline{u}-u-2 \epsilon_{1}|z|^{2}\right)\right) \geq A\left[F\left(H\left(\underline{u}-2 \epsilon_{1}|z|^{2}\right)\right)-F(H(u))\right]=A \epsilon_{0}\left(\epsilon_{1}\right) \\
L V^{ \pm}(z) & \\
\geq & -C_{1}\left(\operatorname{tr}\left(F^{i \bar{j}}\right)+1\right)-F^{i \bar{j}} \partial_{i}\left(u_{t_{\beta}}-\underline{u}_{t_{\beta}}\right) \partial_{\bar{j}}\left(u_{t_{\beta}}-\underline{u}_{t_{\beta}}\right) \\
& +2 F^{i \bar{j}} \partial_{i}\left(u_{t_{\beta}}-\underline{u}_{t_{\beta}}\right) \partial_{\bar{j}}\left(u_{t_{\beta}}-u_{t_{\beta}}\right)+2\left(u_{t_{\beta}}-\underline{u}_{t_{\beta}}\right)\left(\frac{\partial \psi}{\partial t_{\beta}}+F^{i \bar{j}} \frac{\partial^{3} \underline{u}}{\partial z_{i} \partial \bar{z}_{j} \partial t_{\beta}}\right) \\
& +A \epsilon_{0}\left(\epsilon_{1}\right)+A \epsilon_{1} \operatorname{tr}\left(F^{i \bar{j}}\right) \\
\geq & \left(A \epsilon_{0}-C_{1}-2 C\right)+\left(A \epsilon_{1}-C_{1}-2 C\|\underline{u}\|_{C^{3}(\bar{D})}\right) \operatorname{tr}\left(F^{i \bar{j}}\right)
\end{aligned}
$$

where $A \geq\left(C_{1}+2 C\right) / \epsilon_{0}\left(\epsilon_{1}\right)$ and $\epsilon_{0}\left(\epsilon_{1}\right) \leq \epsilon_{1} \leq \epsilon$ and $C=\left(\|u\|_{C^{1}(\bar{D})}+\|\underline{u}\|_{C^{1}(\bar{D})}\right)$.

For $z \in \bar{D}$ near $z=0$, we have $\underline{u}(z)-u(z) \leq 0$. Furthermore, if $z \in \partial D$ we have

$$
V^{ \pm}(z) \leq C|z|^{2}-A \epsilon_{1}|z|^{2} \leq 0, \quad \text { when } A \epsilon_{1} \geq C .
$$


If $z \in D \cap \partial B(0, \delta)$ for some small $\delta>0$ then

$$
V^{ \pm}(z) \leq C(\delta)-A \epsilon_{1} \delta^{2} \leq 0, \quad \text { when } A \epsilon_{1} \geq C(\delta) / \delta^{2} .
$$

where $C, C(\delta)$ is a constant depending only on $\|u\|_{C^{1}(\bar{D})}+\|\underline{u}\|_{C^{1}(\bar{D})}$. Therefore, $V^{ \pm}(z)$ attains its maximum over $\bar{D} \cap \bar{B}(0, \delta)$ at $z=0$ (since $V^{ \pm}(0)=0$ ). Thus

$$
D_{\nu} V^{ \pm}(0) \geq 0
$$

This implies that

$$
\left|D_{\nu T} u\left(z_{0}\right)\right| \leq A+C\left(\|u\|_{C^{1}(\bar{D})}+\|\underline{u}\|_{C^{3}(\bar{D})}+\|\psi\|_{C^{2}(\bar{D})}\right) .
$$

where $A \geq C / \epsilon_{0}\left(\epsilon_{1}\right)+C /\left(\epsilon_{1} \delta^{2}\right)$. So, the proof of the lemma is complete.

LemmA 4.4. Let D, $f \phi$ satisfy conditions of Theorem 1.1. Then

$$
\left|D_{\nu \nu} u(z)\right| \leq C, \quad z \in \partial D
$$

where $C$ is a constant depending only on $f,\|\psi\|_{C^{2}(\bar{D})},\|\phi\|_{C^{4}(\partial D)}, \epsilon$ and $D$.

Proof. Let $z_{0} \in \partial D$ be arbitrary point in $\partial D$. By shift and rotation, we may assume that $z_{0}=0$ and $\rho(z)$ has expression of (4.25)

To prove (4.27), by (4.25) and Lemma 4.3, it suffices to prove

$$
\left|\frac{\partial^{2} u}{\partial x_{n}^{2}}(0)\right| \leq C
$$

Since

$$
4 \sum_{j=1}^{n-1} \frac{\partial^{2} u}{\partial z_{j} \partial \bar{z}_{j}}+\frac{\partial^{2} u}{\partial y_{n}^{2}}+\frac{\partial^{2} u}{\partial x_{n}^{2}}=\Delta u \geq 0
$$

the proof of $(4,28)$ can be reduced to prove

$$
\frac{\partial^{2} u}{\partial x_{n}^{2}}(0) \leq C
$$

In fact, we only need to prove

$$
\frac{\partial^{2} u}{\partial z_{n} \partial \bar{z}_{n}}(0) \leq C
$$

Since

$$
F^{i \bar{j}}(H(u)) \partial_{i \bar{j}}(\underline{u}-u) \geq F(H(\underline{u}))-F(H(u)) \geq \epsilon>0, \quad z \in D
$$

we have that $\underline{u}-u$ attains its maximum over $\bar{D}$ at some point in $\partial D$, but $\underline{u}-u=0$ on $\partial D$, this implies that $D_{\nu}(\underline{u}-u)>0$ on $\partial D$. In particular, $-\frac{\partial}{\partial x_{n}}(\underline{u}-u)(0)>0$.

Let $\Gamma^{\prime}=\left\{\left(\lambda_{1}, \cdots, \lambda_{n-1}\right): \lambda=\left(\lambda_{1}, \cdots, \lambda_{n}\right) \in \Gamma\right\}$ be the projection of $\Gamma$ in $\mathbb{R}^{n-1}$. Since $\lambda(H(\underline{u})) \in \Gamma$, we have $\lambda\left(H(\underline{u})^{\prime}\right) \in \Gamma^{\prime}$. Let

$$
b=-\frac{\partial}{\partial x_{n}}(u-\underline{u})(0)<0 .
$$


Then for $1 \leq \alpha, \beta \leq n-1$, we have

$$
\left.\frac{\partial^{2} u}{\partial z_{\alpha} \partial \bar{z}_{\beta}}(0)=\frac{\partial^{2} \underline{u}}{\partial z_{\alpha} \partial \bar{z}_{\beta}}(0)-\frac{\partial(u-\underline{u})}{\partial x_{n}}(0)\right) \rho_{\alpha \bar{\beta}}(0)=\underline{u}_{\alpha \bar{\beta}}(0)+b \rho_{\alpha \bar{\beta}}(0) \text {. }
$$

It clear that for $t \geq 1, \lambda\left(H(t \underline{u}+b \rho)^{\prime}\right) \in \Gamma^{\prime}, \lambda\left(H(t \underline{u}+b \rho)^{\prime}\right) \notin \Gamma^{\prime}$ when $t$ is very negative, where $H^{\prime}=\left[h_{i \bar{j}}\right]_{1 \leq i, j \leq n-1}$ when $H=\left[h_{i \bar{j}}\right]_{n \times n}$. Let $t_{0}$ be the first $t<1$ so that

$$
\lambda\left(t_{0} H(\underline{u})(0)^{\prime}+b H(\rho)(0)^{\prime}\right) \in \partial \Gamma^{\prime} \Longleftrightarrow t_{0} \lambda\left(H(\underline{u})(0)^{\prime}+\frac{b}{t_{0}} H(\rho)(0)^{\prime}\right) \in \partial \Gamma^{\prime} .
$$

Since $\Gamma^{\prime}$ is a cone, if $t_{0}>0$ then (4.32) implies that $\left(\lambda\left(H(\underline{u})(0)^{\prime}+\frac{b}{t_{0}} H(\rho)(0)^{\prime}\right) \in \Gamma^{\prime}\right.$. Without loss of generality, we may assume that $t_{0}>1 / 2$, otherwise,

$$
\frac{\partial^{2} u}{\partial z_{\alpha} \partial \bar{z}_{\beta}}(0)=\left(1-t_{0}\right) \frac{\partial^{2} \underline{u}}{\partial z_{\alpha} \partial \bar{z}_{\beta}}(0)+t_{0} \frac{\partial^{2} \underline{u}}{\partial z_{\alpha} \partial \bar{z}_{\beta}}(0)+b \rho_{\alpha \bar{\beta}}(0)
$$

with $t_{0} \leq 1 / 2$. From later argument, one can see that this assumption will implies that $u_{n \bar{n}}(0) \leq C$.

Since $b=-\frac{\partial(u-\underline{u})}{\partial x_{n}}(0)<0$, we will show that there is $\eta>0$ independent of $u_{n \bar{n}}\left(z_{0}\right)$ and $z_{0} \in \partial D$ so that

$$
b \geq \frac{b}{t_{0}}+\eta \Longleftrightarrow\left(1-t_{0}\right) \geq t_{0} \eta /|b|=t_{0} \eta /(-b) \geq t_{0} \eta /\left(\|u\|_{1}+\|\underline{u}\|_{1}\right)
$$

where $\|u\|_{1}=\|u\|_{C^{1}(\bar{D})}$. Without loss of generality, we may assume that $\left[\underline{u}_{\alpha \bar{\beta}}(0)+\right.$ $\left.\left(b / t_{0}\right) \rho_{\alpha \bar{\beta}}(0)\right]$ is an $(n-1) \times(n-1)$ diagonal matrix $D_{n-1}\left(\tilde{\lambda}_{1}, \cdots, \tilde{\lambda}_{n-1}\right)$ with $\tilde{\lambda}_{1} \leq$ $\cdots \leq \tilde{\lambda}_{n-1}$.

Since $\Gamma^{\prime}$ is a convex cone in $\mathbb{R}^{n-1}$. If $\Gamma^{\prime}=\mathbb{R}^{n-1}$ then it is easy to handle with. We may assume that $\Gamma^{\prime} \neq \mathbb{R}^{n-1}$. Since $\tilde{\lambda} \in \partial \Gamma^{\prime}$, there is a supporting plane for $\Gamma^{\prime}$. In other words,

$$
\Gamma^{\prime} \subset\left\{\lambda \in \mathbb{R}^{n-1}: \sum_{j=1}^{n-1} \mu_{j}\left(\lambda_{j}^{\prime}-\tilde{\lambda}_{j}\right)>0\right\}, \quad \sum_{j=1}^{n-1} \mu_{j}=1 .
$$

Since $\Gamma^{\prime}$ is symmetric in $\lambda_{j}^{\prime}$ and $\tilde{\lambda}_{1} \leq \cdots \leq \tilde{\lambda}_{n-1}$, it was proved in [8] that one can choose $\mu_{j}$ with $\mu_{1} \geq \mu_{2} \geq \cdots \geq \mu_{n-1} \geq 0$ so that (4.35) holds and $\sum_{j=1}^{n-1} \mu_{j} \tilde{\lambda}_{j}=0$. Therefore, we have $\mu_{1} \geq \mu_{2} \geq \cdots \geq \mu_{n-1} \geq 0$ and

$$
\Gamma^{\prime} \subset\left\{\lambda^{\prime} \in \mathbb{R}^{n-1}: \sum_{j=1}^{n-1} \mu_{j} \lambda_{j}^{\prime}>0\right\}, \quad \sum_{j=1}^{n-1} \mu_{j}=1, \quad \sum_{j=1}^{n-1} \mu_{j} \tilde{\lambda}_{j}=0 .
$$

This implies that $\Gamma \subset\left\{\lambda \in \mathbb{R}^{n}: \sum_{j=1}^{n} \mu_{j} \lambda_{j}>0\right\}$ with $\mu_{n}=0$. Since $f(\lambda(H(\underline{u})(z)) \geq$ $\psi(z)+\epsilon>0$ on $\bar{D}$ and $\underline{u} \in C^{2}(\bar{D})$, we have $\{\lambda(H(\underline{u})(z)): z \in \bar{D}\}$ is a compact subset of $\Gamma$. Thus

$$
\sum_{k=1}^{n} \mu_{k} \underline{u}_{k \bar{k}}(z) \geq \sum_{k=1}^{n} \mu_{k} \lambda_{k}(z) \geq \min \left\{\sum_{k=1}^{n} \mu_{k} \lambda_{k}(z): z \in \bar{D}\right\}=a>0
$$

where $\lambda_{k}(z)$ are eigenvalues of $H(\underline{u})(z)$ with $\lambda_{1}(z) \leq \cdots \leq \lambda_{n}(z)$. 
Let $t_{1}=b / t_{0}$. Then

$$
0=\sum_{k=1}^{n-1} \mu_{k} \tilde{\lambda}_{k}=\operatorname{tr}\left(D\left(\mu_{1}, \cdots, \mu_{n-1}\right)\left(\underline{u}_{\alpha \bar{\beta}}(0)+t_{1} \rho_{\alpha \bar{\beta}}(0)\right)\right)=\sum_{k=1}^{n-1} \mu_{k}\left(\underline{u}_{k \bar{k}}+t_{1} \rho_{k \bar{k}}\right)
$$

Thus

(3.38)

$$
\sum_{k=1}^{n-1} \mu_{k} \rho_{k \bar{k}}(0)=-\frac{1}{t_{1}} \sum_{k=1}^{n-1} \mu_{k} \underline{u}_{k \bar{k}}(0)=\frac{t_{0}}{-b} \sum_{k=1}^{n-1} \mu_{k} \underline{u}_{k \bar{k}}(0) \geq \frac{a}{2\left(\|u\|_{1}+\|\underline{u}\|_{1}\right)}=a_{1}>0 .
$$

Let

$$
d(z)=-\rho(z)+\tau|z|^{2}, \quad z \in D
$$

where $\tau$ is a positive constant with $\tau<a_{1} / 4$; and let

$$
w(z)=\underline{u}(z)+t_{1} \rho(z)+\ell(z) \rho(z)+M d(z)^{2} .
$$

Let

$$
D_{\delta}=D \cap B(0, \delta)
$$

Then, on $\partial D$, we have

$$
u(z)-w(z)=-M \tau^{2}|z|^{4} \leq 0
$$

and, on $D \cap \partial B(0, \delta)$, since $t_{1}<0$ and $\rho(z) \leq 0$, we have

$$
u-w(z) \leq C_{0}\left(\left\|u-\left.\underline{u}\right|_{C(\bar{D})}+\right\| \ell \|_{\infty} \delta\right)-M \tau^{2} \delta^{4} \leq-\frac{M \tau^{2}}{2} \delta^{4}
$$

when $M \geq C_{0}\left(\|u-\underline{u}\|_{C(\bar{D})}+\|\ell\|_{C(\bar{D})} \delta\right) /\left(\delta^{4} \tau^{2}\right)$. As a summary, we have that if $M \geq C_{0}\left(\|u-\underline{u}\|_{C(\bar{D})}+\|\ell\|_{C(\bar{D})} \delta\right) /\left(\delta^{4} \tau^{2}\right)$ with small $\delta>0$ fixed, then

$$
u \leq w, \quad \text { on } \partial D_{\delta}
$$

Let $\left(T_{1}(z), \cdots, T_{n-1}\right)$ be an orthonormal basis for holomorphic tangent space of level hypersurface $d(w)=d(z)$ at $z$, we choose $T_{j}$ are $C^{1}$ and $T_{j}\left(0, z_{n}\right)=e_{j}$, where $e_{j}=(0, \cdots, 0,1,0, \cdots, 0)$. Let $\mu_{n}=0$, and $T_{n}=\partial d /|\partial d|$. Then we define

$$
\Lambda=\sum_{k=1}^{n} \mu_{k} \bar{T}_{k i} T_{k j} \frac{\partial^{2}}{\partial z_{i} \partial \bar{z}_{j}}
$$

Thus,

$$
\Lambda d(z)^{2}=2 d \Lambda d(z)+2 \sum_{k=1}^{n-1} \mu_{k} \bar{T}_{k i} T_{k j} \partial_{i} d(z) \partial_{\bar{j}} d(z)=2 d(z) \Lambda d(z) \leq-\frac{a_{1}}{2} d(z)
$$

since

$$
\Lambda d(z)=\Lambda\left(0, z_{n}\right) d(z)+\left(\Lambda-\Lambda\left(0, z_{n}\right)\right) d(z) \leq-a_{1}+\tau+C|\rho(z)| \leq-\frac{a_{1}}{4} .
$$


where $|\rho(z)| \leq a_{1} /(4 C)$ with $C=\left\|\Lambda(z) d(z)-\Lambda\left(0, z_{n}\right) d(z)\right\|_{\infty}+1$.

Now we claim that $\lambda\left(H(w)(z)^{\prime}\right) \notin \Gamma^{\prime}$ for $z \in \bar{D}_{\delta}$. Since

$$
H(w)=H\left(\underline{u}+t_{1} \rho\right)+\ell H(\rho)+\partial \ell \otimes \bar{\partial} \rho+\partial \rho \otimes \bar{\partial} \ell+M H\left(d^{2}\right)
$$

and $\Lambda(0)\left(\underline{u}+t_{1} \rho\right)(0)=0$ we have

$$
\begin{gathered}
\Lambda\left(\underline{u}+t_{1} \rho\right)(z)=\sum_{j=1}^{n}\left(A_{j} z_{j}+\bar{A}_{j} z_{j}\right)+O_{1}\left(|z|^{2}\right)=L_{1}(z)+O_{1}\left(|z|^{2}\right) \\
\ell(z) \Lambda \rho(z)=\ell(z) \Lambda \rho(0)+O_{2}\left(|z|^{2}\right), \quad(\Lambda \rho)(0) \in\left(-C,-a_{1}\right]
\end{gathered}
$$

and

$$
\tau \sum_{k=1}^{n} \mu_{k} \bar{T}_{k i} T_{k j}\left(\ell_{i} z_{j}+\ell_{\bar{j}} \bar{z}_{i}\right)=\tau \sum_{k=1}^{n} \mu_{k}\left(\ell_{i} z_{i}+\ell_{\bar{i}} \bar{z}_{i}\right)+O_{3}\left(|z|^{2}\right)
$$

Therefore,

$$
\begin{aligned}
\Lambda w= & \Lambda\left(\underline{u}+t_{1} \rho\right)+\ell(z) \Lambda \rho(z)+\sum_{k=1}^{n} \mu_{k} \bar{T}_{k i} T_{k j}\left(\ell_{i} \bar{\partial}_{j} \rho+\ell_{\bar{j}} \partial_{i} \rho\right)+M \Lambda d^{2} \\
\leq & L_{1}(z)+O_{1}\left(|z|^{2}\right)+(\Lambda \rho)(0) \ell(z)+O_{2}\left(|z|^{2}\right) \\
& +\tau \sum_{k=1}^{n} \mu_{k} \bar{T}_{k i} T_{k j}\left(\ell_{i} z_{j}+\ell_{\bar{j}} \bar{z}_{i}\right)-\frac{M a_{1}}{2} d(z) \\
= & L_{1}(z)+O_{1}\left(|z|^{2}\right)+(\Lambda \rho)(0) \ell(z)+O_{2}\left(|z|^{2}\right)+\tau O_{3}\left(|z|^{2}\right) \\
& +\tau \sum_{k=1}^{n} \mu_{k}\left(\ell_{k} z_{k}+\ell_{\bar{k}} \bar{z}_{k}\right)-\frac{M a_{1}}{2} d(z) \\
= & \sum_{k=1}^{n}\left(A_{k}+\ell_{k}\left((\Lambda \rho)(0)-\tau \mu_{k}\right)\right) z_{k}+\left(\bar{A}_{k}+\ell_{\bar{k}}\left((\Lambda \rho)(0)-\tau \mu_{k}\right)\right) \bar{z}_{k} \\
& +O\left(|z|^{2}\right)-\frac{M a_{1}}{2} d(z) \\
= & O\left(|z|^{2}\right)-\frac{M a}{2} d(z) \\
\leq & -\frac{M a}{4} d(z)
\end{aligned}
$$

by choosing $\ell_{k}$ and $M>0$ so that

$$
\ell_{k}=-A_{k} /\left((\Lambda \rho)(0)-\tau \mu_{k}\right), \quad \text { and } O\left(|z|^{2}\right) \leq \frac{M a_{1}}{4}|z|^{2} .
$$

Let $\lambda_{k}(w)$ are all eigenvalues of $H(w)$ at $z$ with $\lambda_{1}(w) \leq \cdots \leq \lambda_{n}(w)$. Then by Proposition 2.4 with $B=\operatorname{Diag}\left(\mu_{1}, \cdots, \mu_{n-1}, 0\right)$

$$
\sum_{k=1}^{n-1} \mu_{k} \lambda_{k}(w) \leq \operatorname{tr}(H(w)(z) B)=\Lambda w(z)<0
$$


Thus $\lambda\left(H(w)^{\prime}\right) \notin \Gamma^{\prime}$.

Let

$$
\tilde{\Gamma}=\{\lambda \in \Gamma: f(\lambda) \geq \min \{\psi(z): z \in \bar{D}\}>0\}
$$

and let

$$
X=\left\{\lambda \in \mathbb{R}^{n}: \lambda^{\prime} \in \mathbb{R}^{n-1} \backslash \Gamma^{\prime}:|\lambda| \leq\|w\|_{C^{2}(\bar{D})}+1\right\} .
$$

Then for any $\lambda \in \partial \Gamma$ by Assumption (1.10), we have $f(\lambda) \leq \psi^{0}<\min \{\psi(z): z \in \bar{D}\}$. Since $f$ is continuous on compact set $X$, there is $\eta>0$ such that if $\lambda \in X$ and $(\eta, \cdots, \eta)+\lambda \in \Gamma$ then

$$
f((\eta, \cdots, \eta)+\lambda)<\psi_{0}=\min \{\psi(z): z \in \bar{D}\}
$$

Let

$$
v(z)=w(z)+\eta\left(|z|^{2}-\frac{1}{C_{0}} x_{n}\right)
$$

where $C_{0}>0$ be chosen so that $|z|^{2}-\frac{1}{C_{0}} x_{n} \geq 0$ on $\partial D_{\delta}$. Then $u(z) \leq v(z)$ on $\partial D_{\delta}$. Since $\lambda(H(v))=\left(\lambda\left(H(w)+\eta I_{n}\right) \notin \tilde{\Gamma}\right.$ by (4.48), (4.46), (4.47) and $\lambda\left(H(w)^{\prime}\right) \notin \Gamma^{\prime}$. By Maximum Principle, i.e., Lemma 2.1, we have $u(z)-v(z) \leq 0$ on $\bar{D}_{\delta}$. Since $u(0)-v(0)=0$, we have

$$
\begin{gathered}
-\frac{\partial}{\partial x_{n}}(u-v)(0) \geq 0 \Longleftrightarrow-\frac{\partial(u-\underline{u})}{\partial x_{n}}(0) \geq \frac{\eta}{C_{0}}+t_{1} \Longleftrightarrow b \geq \frac{\eta}{C_{0}}+\frac{b}{t_{0}} \Longleftrightarrow \\
\Longleftrightarrow\left(1-t_{0}\right) \geq \frac{\eta t_{0}}{C_{0}|b|}
\end{gathered}
$$

Therefore

$$
\begin{aligned}
\frac{\partial^{2} u}{\partial z_{\alpha} \partial \bar{z}_{\beta}}(0) & =\frac{\partial^{2} \underline{u}}{\partial z_{\alpha} \partial \bar{z}_{\beta}}(0)-\frac{\partial(u-\underline{u})}{\partial x_{n}}(0) \rho_{\alpha \bar{\beta}}(0) \\
& =\left(1-t_{0}\right) \frac{\partial^{2} \underline{u}}{\partial z_{\alpha} \partial \bar{z}_{\beta}}(0)+t_{0}\left[\frac{\partial^{2} \underline{u}}{\partial z_{\alpha} \partial \bar{z}_{\beta}}(0)+t_{1} \rho_{\alpha \bar{\beta}}(0)\right]
\end{aligned}
$$

Thus

$$
H(u)(0)=E_{0}+\left[\begin{array}{cc}
\left(1-t_{0}\right) \underline{u}_{\alpha \bar{\beta}}(0) & O(1) \\
O(1)^{*} & u_{n \bar{n}}(0)-M_{1}
\end{array}\right]
$$

where $\lambda\left(E_{0}\right) \in \bar{\Gamma}$ for some fixed $M_{1}$. Therefore, since $\lambda(H(\underline{u})(0)) \in \Gamma$ and

$$
\begin{aligned}
\psi(0) & =F(H(u)(0)) \geq t_{0} F\left(E_{0} / t_{0}\right)+\left(1-t_{0}\right) F\left(\left(\left[\begin{array}{cc}
\underline{u}_{\alpha \bar{\beta}} & O(1) \\
O(1)^{*} & \left(u_{n \bar{n}}(0)-M_{1} 0 /\left(1-t_{0}\right)\right.
\end{array}\right]\right)\right. \\
& \geq\left(1-t_{0}\right) F\left(\left(\left[\begin{array}{cc}
\underline{u}_{\alpha \bar{\beta}} & O(1) \\
O(1)^{*} & \left(u_{n \bar{n}}(0)-M_{1}\right) /\left(1-t_{0}\right)
\end{array}\right]\right)\right.
\end{aligned}
$$

Notices that $\left(1-t_{0}\right) \geq \frac{\eta}{2 C_{0}} /\left(\|u\|_{1}+\|\underline{u}\|_{1}\right)>0$ and Lemma 2.2 and Condition (1.11), we have

$$
u_{n \bar{n}}(0) \leq C \text {. }
$$


where $C$ is depending only on $\|\underline{u}\|_{C^{3}(\bar{D})},\|\psi\|_{C^{2}(\bar{D})}, \epsilon,\|\phi\|_{C^{4}(\partial D)}$ and $\Gamma$. Thus, the proof of Lemma 4.4 is complete.

We now are ready to prove Theorems $1.1-1.3$.

Proof of Theorem 1.1. Under the assumption of Theorem 1.1, we have that the all assumptions of Lemmas 4.1-4.4 hold. Combining the results of Lemmas 4.1-4.4, we have $a$ priori estimate on the admissible solution $u$ of (1.14) satisfying the estimate:

$$
\|u\|_{C^{2}(\bar{D})} \leq C\left(\epsilon, f,\|\phi\|_{C^{4}(\partial D)},\|\psi\|_{C^{2}(\bar{D})}\right)<\infty .
$$

The results in [6] and [23] and arguments in [8] and [7] implies that the Dirichlet problem (1.14) has a unique solution $u \in C^{\infty}(\bar{D})$ with $\lambda(H(u)) \in \Gamma$ on $D$. Thus, the proof of Theorem 1.1 is complete.

Proof of Theorem 1.2. The assumptions of Theorem 1.2 imply that the all assumptions of Theorems 3.1 and 3.2 hold. For any $\phi \in C^{\infty}(\bar{D})$, Theorem 3.2 implies that there is $\underline{u} \in C^{\infty}(\bar{D})$ so that $\lambda(H(\underline{u})) \in \Gamma$ for all $z \in \bar{D}$ and

$$
f(\lambda(H(\underline{u})(z)) \geq \psi(z)+1, \quad z \in \bar{D} .
$$

Theorem 1.1 gives the result of Theorem 1.2.

Proof of Theorem 1.3. The proof of the sufficient condition of Theorem 1.3 is a consequence of Proposition 3.3 and Theorem 1.2. The proof of the necessary condition can be followed directly from an argument in [8], we omit the detail here.

\section{REFERENCES}

[1] E. Bedford, Survey of Pluri-Potential Theory, Several Complex Variables, pp. 49-97, Math. Notes 38, Princeton University Press, NJ, 1993.

[2] E. Bedford And B.A. TAYloR, The Dirichlet problem for a complex Monge-Ampère equation, Invent. Math., 37 (1976), pp. 1-44.

[3] E. Bedford And J. Fornaess, Counter examples to regularity for complex Monge-Ampère equation, Invent. Math., 50 (1979), pp. 129-134.

[4] D. Bunns, Curvatures of Monge-Ampère foliations and parabolic manifolds, Ann. Math., 115 (1982), pp. 349-373.

[5] J. Bland, The Einstein-Kähler metric on $\left\{|z|^{2}+|w|^{2 p}<1\right\}$, Mich. Math. J., 33 (1986), pp. 209-220.

[6] L. Caffarelli, L. Nirenberg and J. Spruck, The Dirichlet problem for nonlinear secondorder elliptic equations, I: Monge-Ampère equations, Comm. Pure Appl. Math., 37 (1984), pp. 369-402.

[7] L. Caffarelli, J. J. Kohn, L. Nirenberg and J. Spruck, The Dirichlet problem for nonlinear second-order elliptic equations, II: complex Monge-Ampère, and uniformly elliptic equations, Comm. on Pure and Appl. Math., (1985), pp. 209-252.

[8] L. Caffarelli, L. Nirenberg And J. Spruck, The Dirichlet problem for nonlinear secondorder elliptic equations, III: Functions of the eigenvalues of the Hessian, Acta Math., (1986), pp. 261-301.

[9] S. Y. Cheng And S. T. YAu, On the existence of a complex Kähler metric on non-compact complex manifolds and the regularity of Fefferman's equation, Comm. Pure Appl. Math., 33 (1980), pp. 507-544.

[10] S. Y. Cheng And S. T. YAU, On the regularity of the Monge-Ampère equation $\operatorname{det}\left(\partial^{2} u / \partial x_{i} \partial x_{j}\right)=F(x, u)$, Comm. Pure Appl. Math., 30 (1977), pp. 41-68.

[11] J.-P. Demailly, Measures de Monge-Ampère et mesures pluriharmoniques, Math. Z., 194 (1987), pp. 519-564.

[12] C. Fefferman, Monge-Ampère equations, the Bergman kernel, and geometry of pseudoconvex domains, Ann. of Math., 103 (1976), pp. 395-416.

[13] J. E. Fornaess And N. Sibony, Construction of plurisubharmonic functions on weakly pseudoconvex domains, Duke Math. J., 58 (1989), pp. 633-655. 
[14] L. Garding, An inequality for hyperbolic polynomials, J. Math. Mech., 8 (1959), pp. 957-965.

[15] B. GuAn, The Dirichlet problem for complex Monge-Ampère equations and Regularity of the Green's function, Comm. in Analysis and Geometry, 6 (1998), pp. 687-703.

[16] B. Guan And P. Guan, Hypersurfaces with prescribed curvatures, Ann. of Math., 156 (2002), pp. 655-674.

[17] B. GuAn And Y. LI, On Weyl problems with nonnegative Gauss curvature, JDG., 39 (1994), pp. 331-342.

[18] P. Guan And X-N. Ma, The Christoffel-Minkowski Problem I: Converity of solutions of Hessian equation, preprint.

[19] P. GuAn AND C. S. Lin AND X. MA, The Christoffel-Minkowski Problem II, preprint.

[20] P. Guan, N.S. Trudinger and X-J. Wang, On the Dirichlet problem for degerate MongeAmpère equations, Acta Math., 182 (199), pp. 87-104.

[21] J. J. Kohn And L. NirenberG, Noncoercive boundary value problems, Comm. Pure Appl. Math., 18 (1965), pp. 443-492.

[22] S. KolodzieJ, The complex Monge-Ampère equations, Acta Math., 180 (1998), pp. 69-117.

[23] N. V. Krylov, Boundeddly inhomogeneous elliptic and parabolic equations in a domain, Izv. Akad. Nauk SSSR, 47 (1983), pp. 75-108.

[24] J. Lee And R. Melrose, Boundary behavior of the complex Monge-Ampère equation, Acta Math., (1982), pp. 159-192.

[25] L. LEMPERT, Solving the degenerate Monge-Ampère equation with one concentrated singularity, Math. Ann., 263 (1983), pp. 515-532.

[26] S-Y. LI, On Dirichlet problems for complex Monge-Ampère equations in weakly pseudoconvex domains, Sumbitted for publication.

[27] S-Y. LI, The Neumann Problem for Complex Monge-Ampere equation, Indiana University J. of Mathematics, 44 (1995), pp. 1099-1122.

[28] S-Y. LI, On oblique boundary value problem for Monge-Ampère equation, Pacific J. of Math., 190 (1999), pp. 155-172.

[29] P. L. Lions, N. S. Trudinger, And J. I. E. URbas, The Neumann problem for equations of Monge-Ampére type, Comm. Pure Appl. Math., 39 (1986), pp. 539-563.

[30] H-S. LUK AND STEPhEN YAU, Counterexample to boundary regularity of a strongly pseudoconvex CR submanifold: an addendum to the paper of Harvey-Lawson, Ann. of Math., (2) 148:3 (1998), pp. 1153-1154.

[31] M. Marcus, An eigenvalue inequality for product of normal matrices, Amer. Math. Monthly, 63 (1956), pp. 173-174.

[32] Y.-T. Siu, Pseudoconvexity and the problem of Levi, Bull. Amer. Math. Soc., 84 (1978), pp. $481-512$.

[33] Y.-T. Siu, The Levi Problem, Several Complex Variables, Proc. Sympos. Pure Math., XXXX (1975), AMS Providence, R. I., 1977, pp.45-48.

[34] J. URBAS, On the expansion of starshaped hypersurfaces by symmetric functions of their principle curvature, Math. Z., 205 (1990), pp. 355-372.

[35] S. T. YAU, On the Ricci curvature of a compact Kähler manifold and the complex MongeAmpère equation, Comm. in Pure and Appl. Math., 31 (1978), pp. 339-411. 